\title{
Neutrosophic Function with NNs for Analyzing and Expressing Anisotropy Characteristic and Scale Effect of Joint Surface Roughness
}

\author{
Rui Yong $\mathbb{D}^{1},{ }^{1}$ Leiyu Gu $\mathbb{D}^{2},{ }^{2}$ Jun Ye $\mathbb{D}^{1},{ }^{1}$ Shi-Gui Du $\mathbb{D}^{1},{ }^{1}$ Man Huang, \\ Gaojian Hu, ${ }^{1}$ and Jie Liu ${ }^{1}$ \\ ${ }^{1}$ Center of Rock Mechanics and Geohazards, Shaoxing University, 508 Huancheng West Road, Shaoxing, \\ Zhejiang Province 312000, China \\ ${ }^{2}$ Shanghai Institute of Disaster Prevention and Relief, 1239 Siping Road, Shanghai, 200433, China \\ Correspondence should be addressed to Leiyu Gu; leiyugu@126.com
}

Received 17 November 2018; Accepted 25 March 2019; Published 14 April 2019

Academic Editor: Mijia Yang

Copyright (c) 2019 Rui Yong et al. This is an open access article distributed under the Creative Commons Attribution License, which permits unrestricted use, distribution, and reproduction in any medium, provided the original work is properly cited.

\begin{abstract}
The shear behavior of rock mass significantly depends upon the surface roughness of rock joints which is generally characterized by the anisotropy characteristic and the scale effect. The large-scale natural rock joint surfaces, at Qingshi Town, southeast of Changshan County, Zhejiang Province, China, were used as a case study to analyze the roughness characteristics. A statistical assessment of joint roughness coefficient $(J R C)$ indicated the roughness anisotropy of different sized rock joints. The lower limit $\left(J R C_{\text {mean }}-\sigma\right)$ was regarded as the determinate information, and the difference between lower and upper limits represented indeterminate information. The neutrosophic number $(\mathrm{NN})$ was calculated to express the various $J R C$ values. The parametric equations for $J R C$ anisotropic ellipse were presented based on the $J R C$ statistical assessment of joint profiles of various orientations. The $J R C$ values of different sized joint samples were then quantitatively described by the neutrosophic function. Finally, a neutrosophic parameter $\psi$ for evaluating the scale effect on the surface roughness anisotropy was introduced using the ratio of maximum directional roughness to minimum directional roughness. The case study indicates that the proposed method has the superiority in moving forward from subjective assessment to quantitative and objective analysis on anisotropy characteristic and scale effect of joint surface roughness.
\end{abstract}

\section{Introduction}

Joint roughness is one of the most important parameters for understanding the shear behavior of rock joints [1-5]. Yet the irregularity or roughness of rock surfaces is difficult to estimate. Thus, during the past few decades, considerable effort has been devoted to estimating the roughness properties of rock joint surfaces. Since Barton [6] first introduced the joint roughness coefficient $(J R C)$ for quantifying rock joint roughness, various roughness parameters have been established for roughness characterization [7-12]. The scale effect on surface roughness has been proven to be an inherent property of rock joints. Consequently, a large number of researchers have attempted to determine the scale dependency of joint surface roughness by relating the roughness parameters to the joint sample size. Du et al. [13] found that the negative scale effect exists in rock joint roughness by statistical analysis of asperity amplitudes. Furthermore, anisotropy is everywhere in rock engineering, and the joint roughness varies directionally, which is an important source of anisotropic behavior of rock joints. Chen et al. [14] used geological statistics to analyze the anisotropy and the scale effect of rock joints using the sill value and the range of variogram. The statistical analysis of the directional $J R C$ values of different sized rock joint samples may disclose the scale effect of joint roughness. Fardin et al. [15] investigated the scale effect on the surface roughness of rock joints using fractal concepts. The fractal parameters remained almost constant when the sampling windows were larger than the stationarity threshold. Yong et al. [16] analyzed the sampling problem in studying the scale effect of rock 
joints and proposed a sampling method based on a systematic sampling technique for quantitatively evaluating roughness characteristics and representativeness of different sized rock joints. However, the roughness characteristics are difficult to quantify because of the irregularity and inconsistency of joint roughness of different sized samples in various measurement orientations. Moreover, none of the previous studies considered the scale effect on the surface roughness anisotropy of rock joints.

The real world consists of determinate and/or indeterminate information. A neutrosophic number (NN) presented by Smarandache [17-19] seems appropriate for expressing them because a NN $(z=a+b I)$ consists of a determinate part $a$ and an indeterminate part $b I$ for $a, b \in R$, where the symbol $I$ denotes indeterminacy and $R$ represents real numbers. Recently, Ye [20] developed a bidirectional projection model of NNs for a multiple attribute group decision-making problem. Then, Ye [21] proposed a deneutrosophication method and a possibility degree ranking method for neutrosophic numbers and applied these methods to a group decisionmaking problem under a NN environment. Kong et al. [22] introduced a cosine similarity measure of NNs and applied it to the misfire fault diagnosis of gasoline engine. Further, Ye [23] presented an exponential similarity measure of NNs for the fault diagnosis of steam turbine in a NN environment. To express indeterminate functions in indeterminate problems, Smarandache [24] introduced the interval function (thick function), which is defined as a neutrosophic function $h$ : $R \longrightarrow H(R)$, where $R$ represents all real numbers and $H(R)$ is all interval functions, denoted by the form of an interval function $h(x)=\left[h_{1}(x), h_{2}(x)\right]$ for $x \in R$. Smarandache's neutrosophic function, however, cannot express and deal with functions involving NNs. Ye et al. [25] expressed the joint roughness coefficient and the shear strength in rock mechanics by means of neutrosophic functions (interval functions). It is necessary to introduce new neutrosophic functions containing NNs for statistical assessment of joint roughness, containing both determinate information and indeterminate information. Consequently, $\mathrm{NN}$ is appropriate for expressing various $J R C$ values, because it consists of determinate and indeterminate parts.

The objectives of this paper are as follows: (1) presenting some basic operations of NNs and new neutrosophic function with NNs; (2) developing the parametric equations for JRC anisotropic ellipse to characterize the joint profiles in various orientations; (3) presenting a neutrosophic function with NNs to describe the JRC values of different sized joint samples; and (4) developing a neutrosophic parameter $\psi$ to evaluate the scale effect on the surface roughness anisotropy using the ratio of maximum directional roughness to minimum directional roughness.

\section{NNs and Neutrosophic Functions}

2.1. Some Basic Operations of NNs. In indeterminate environments, Smarandache [17-19] presented the concept of NN. It consists of a determinate part $a$ and an indeterminate part $b I$. Its mathematical expression is $z=a+b I$ for $a, b \in R$, where $I$ is indeterminacy and $R$ represents all real numbers. It has been successfully applied to represent determinate and/or indeterminate information in real problems.

When $\mathrm{NN}$ is considered as $z=2+2 I$, its determinate value is 2 and its indeterminate value is $2 I$. In application, a possible interval range of indeterminacy $I$ is often specified to satisfy concrete application requirements. For example, if the indeterminacy $I$ is considered to fall within an possible interval $[0,0.1]$, then it is equivalent to $z=[2,2.2]$, where $z$ is within the interval $[2,2.2]$. If $I \in[0.1,0.2]$ is the interval range, then $z=[2.2,2.4]$.

Yet, $z=a+b I$ may be a possible interval number, where $z=\left[a+b I^{L}, a+b I^{U}\right]$ for $z \in Z$ (Z is all NNs) and $I \epsilon$ $\left[I^{L}, I^{U}\right]$. Specially, if $b I=0$ for the best case, then $z$ equals the determinate part $z=a$. But if $a=0$ is the worst case, then $z$ is the same as the indeterminate part $z=b I$.

For two NNs $z_{1}=a_{1}+b_{1} I$ and $z_{2}=a_{2}+b_{2} I$ for $z_{1}, z_{2} \in$ $\mathrm{Z}$ and $I \in\left[I^{L}, I^{U}\right]$, the basic operational laws are derived as follows:

$$
\begin{aligned}
& z_{1}+z_{2}=a_{1}+a_{2}+\left(b_{1}+b_{2}\right) I \\
& =\left[a_{1}+a_{2}+b_{1} I^{L}+b_{2} I^{L}, a_{1}+a_{2}+b_{1} I^{U}+b_{2} I^{U}\right] \text {; } \\
& z_{1}-z_{2}=a_{1}-a_{2}+\left(b_{1}-b_{2}\right) I \\
& =\left[a_{1}-a_{2}+b_{1} I^{L}-b_{2} I^{L}, a_{1}-a_{2}+b_{1} I^{U}-b_{2} I^{U}\right] \text {; } \\
& z_{1} \times z_{2}=a_{1} a_{2}+\left(a_{1} b_{2}+a_{2} b_{1}\right) I+b_{1} b_{2} I^{2} \\
& =\left[\begin{array}{c}
\min \left(\begin{array}{c}
\left(a_{1}+b_{1} I^{L}\right)\left(a_{2}+b_{2} I^{L}\right),\left(a_{1}+b_{1} I^{L}\right)\left(a_{2}+b_{2} I^{U}\right), \\
\left(a_{1}+b_{1} I^{U}\right)\left(a_{2}+b_{2} I^{L}\right),\left(a_{1}+b_{1} I^{U}\right)\left(a_{2}+b_{2} I^{U}\right)
\end{array}\right), \\
\max \left(\begin{array}{c}
\left(a_{1}+b_{1} I^{L}\right)\left(a_{2}+b_{2} I^{L}\right),\left(a_{1}+b_{1} I^{L}\right)\left(a_{2}+b_{2} I^{U}\right), \\
\left(a_{1}+b_{1} I^{U}\right)\left(a_{2}+b_{2} I^{L}\right),\left(a_{1}+b_{1} I^{U}\right)\left(a_{2}+b_{2} I^{U}\right)
\end{array}\right)
\end{array}\right] ; \\
& \frac{z_{1}}{z_{2}}=\frac{a_{1}+b_{1} I}{a_{2}+b_{2} I}=\frac{\left[a_{1}+b_{1} I^{L}, a_{1}+b_{1} I^{U}\right]}{\left[a_{2}+b_{2} I^{L}, a_{2}+b_{2} I^{U}\right]} \\
& =\left[\begin{array}{l}
\min \left(\frac{a_{1}+b_{1} I^{L}}{a_{2}+b_{2} I^{U}}, \frac{a_{1}+b_{1} I^{L}}{a_{2}+b_{2} I^{L}}, \frac{a_{1}+b_{1} I^{U}}{a_{2}+b_{2} I^{U}}, \frac{a_{1}+b_{1} I^{U}}{a_{2}+b_{2} I^{L}}\right) \\
\max \left(\frac{a_{1}+b_{1} I^{L}}{a_{2}+b_{2} I^{U}}, \frac{a_{1}+b_{1} I^{L}}{a_{2}+b_{2} I^{L}}, \frac{a_{1}+b_{1} I^{U}}{a_{2}+b_{2} I^{U}}, \frac{a_{1}+b_{1} I^{U}}{a_{2}+b_{2} I^{L}}\right)
\end{array}\right] .
\end{aligned}
$$

\section{Data Acquisition}

A $141.4 \mathrm{~cm} \times 141.4 \mathrm{~cm}$ joint surface sample was collected from a large sized, slightly weathered, slate rock surface with an orientation of N40W, 35NW at Qingshi Town, southeast of Changshan County, Zhejiang Province, China. The grayish-green colored slate rock is foliated, very finegrained, and formed by the metamorphosis of intermediate tuff, and the joint surface is relatively smooth and planar. The surface profiles were measured in 25 different orientations at an interval of $15^{\circ}$ (Figure 1). A simple mechanical hand profilograph (Figure 2) was employed to measure the rock joint profiles. The profilograph consists of feeler, drawing pen, balance block, fixed board, bubble levels, and drawing paper. It is easy to operate and correctly records a large number of joint traces with minimal cost. In this study, the number of measured roughness profiles with different orientations and sample sizes was 19,616. These recorded joint profiles were scanned by a large format scanner. On the basis of pixel 


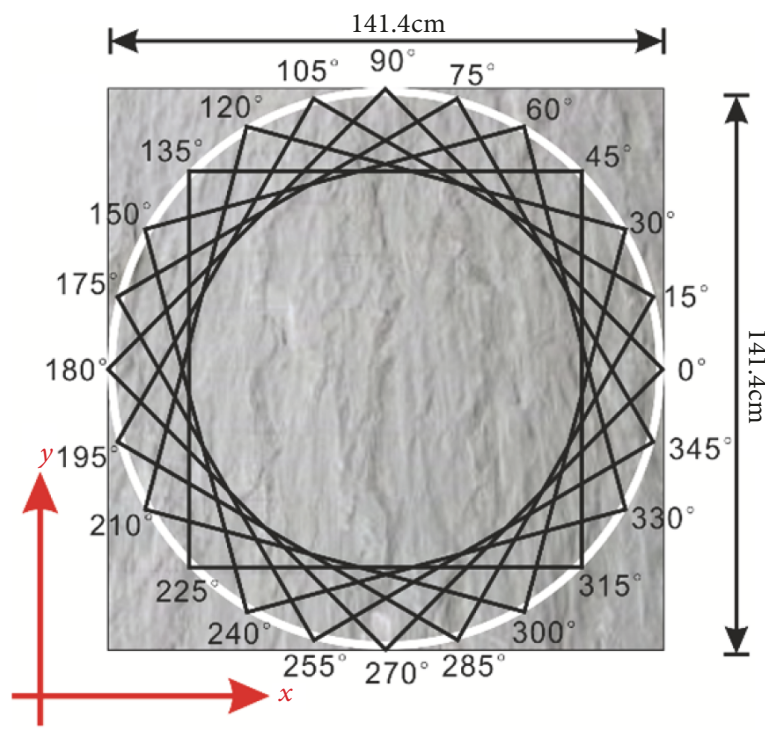

(a)

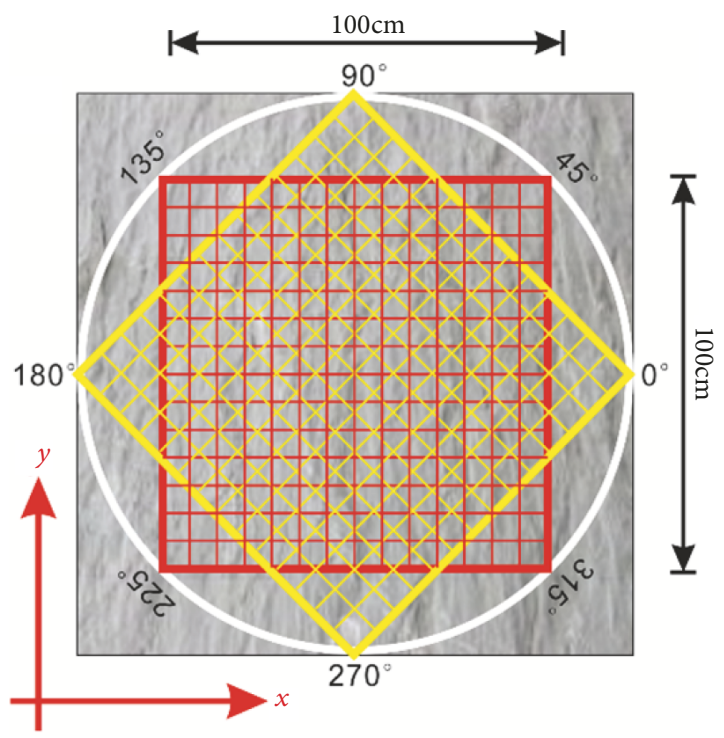

(b)

Figure 1: Arrangement of measuring profiles on the natural slate joint surface. (a) Measurement orientation; (b) examples of the arrangement of the test profiles in the orientations of $0^{\circ}, 45^{\circ}, 90^{\circ}, 135^{\circ}, 180^{\circ}, 225^{\circ}, 270^{\circ}$, and $315^{\circ}$.

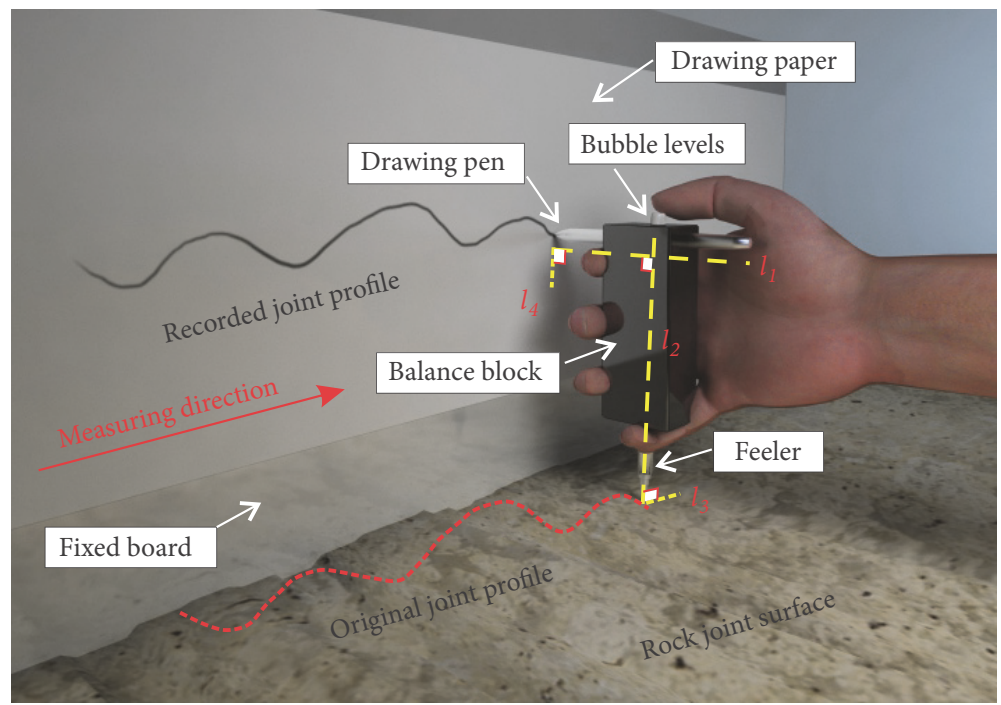

FIGURE 2: Mechanical hand profilograph used for measuring the profiles of rock joints.

analysis, the joint profiles were then digitized with a sampling interval of $0.5 \mathrm{~mm}$, which is fairly often used in previous studies on joint roughness determination [26].

Many studies regarding JRC evaluation have been carried out over the past three decades. The most commonly used methods include visual comparison, root mean square method $\left(Z_{2}\right)$, roughness profile index method $\left(R_{p}\right)$, and fractal approaches $(D)$. However, none of these studies considered the combined effects of shear direction, the scale of joint surfaces, the inclination angle, and the amplitudes of asperities in joint roughness calculations. To overcome these shortcomings, Zhang et al. [27] developed a logistic function between JRC and the ratio of asperity amplitude to profile length. The inclination angle, the amplitude of asperities, and their directions were considered in this method. This procedure was proved to be accurate and efficient in JRC evaluation via the comparisons with different kinds of test results. Consequently, it was used in this study to calculate the $J R C$ values for different sized and orientated profiles. It is briefly introduced as follows.

First, an arbitrary horizontal reference line was assumed to pass through the digitized profile. The mean vertical distance between the reference line and the points along the roughness profile was calculated by

$$
h_{a v}=\frac{\int_{x=0}^{x=L}|y| d x}{L}=\sum_{i=1}^{N} \frac{\left|y_{i+1}+y_{i}\right|\left(x_{i+1}-x_{i}\right)}{2 L}
$$


where $L$ is the length of a digitized roughness profile and $x$ and $y$ are the horizontal and vertical coordinates of the points on the profile, respectively.

Those profile segments whose dip-direction is opposite to the shear direction were significant in determining joint roughness in the direction of shearing. The modified root mean square $Z_{2}^{\prime}$ taking into account the positive dilation angles was determined by

$$
\begin{aligned}
Z_{2}^{\prime} & =\sqrt{\frac{1}{L} \int_{x=0}^{x=L}\left(\max \left(0, \frac{d y}{d x}\right)\right)^{2} d x} \\
& =\left[\sum_{i=1}^{M} \frac{\left(\max \left(0, y_{i+1}-y_{i}\right)\right)^{2}}{\left(x_{i+1}-x_{i}\right) L}\right]^{1 / 2}
\end{aligned}
$$

A roughness index $\lambda$ was introduced by Zhang et al. [27] and written as

$$
\lambda=\left(\frac{h}{L}\right)^{\alpha} \cdot\left(Z_{2}^{\prime}\right)^{1-\alpha}, \quad \alpha=\frac{1}{3}
$$

The JRC value was then calculated based on the following logistic correlation with $\lambda$, as

$$
J R C=\frac{40}{1+e^{-20 \lambda}}-20
$$

This procedure was repeated to determine the JRC evaluation for all profiles.

\section{Neutrosophic Function with NNs for Anisotropy Characteristics of Joint Surface Roughness}

The $J R C$ values of joint samples in different orientations were calculated and displayed on the polar plots. For example, Figure 3 shows the $J R C$ values of $10 \mathrm{~cm}$ joint samples with various orientations from $0^{\circ}$ to $345^{\circ}$ at a $15^{\circ}$ interval. As can be observed, the $J R C$ values in some orientations (e.g., $0^{\circ}$ and $180^{\circ}$ ) were greater, while the values in the orientations of $90^{\circ}$ and $270^{\circ}$ were relatively smaller. The $J R C$ values in all orientations were randomly distributed in a range from 0 to 20 . The $J R C$ distributions of $10 \mathrm{~cm}$ joint samples in the orientations of $0^{\circ}, 90^{\circ}, 180^{\circ}$, and $270^{\circ}$ are illustrated in Figure 4. These frequency distributions can be represented graphically by the characteristic bell-shaped curves, which are close to the "Normal Distribution" or the "Right-skewed Distribution." The mean value $J R C_{\text {mean }}$ and the standard deviation $\sigma$ of $J R C$ values in the $0^{\circ}$ orientation were 10.536 and 2.233. For those joint samples in the $180^{\circ}$ orientation, $J R C_{\text {mean }}$ and $\sigma$ were 9.850 and 2.144 , respectively. The $J R C$ mean values in the $90^{\circ}$ and $270^{\circ}$ orientations were 7.047 and 6.802 , respectively, and the standard deviations were 2.405 and 2.117.

A low standard deviation indicates that the data points tend to approximate the mean value. Here, the $J R C$ values in a range from $J R C_{\text {mean }}-\sigma$ (lower limit) to $J R C_{\text {mean }}+\sigma$ (upper limit) were chosen to describe the roughness anisotropy characteristics. As shown in Figure 4, a total of $68.62 \%$,

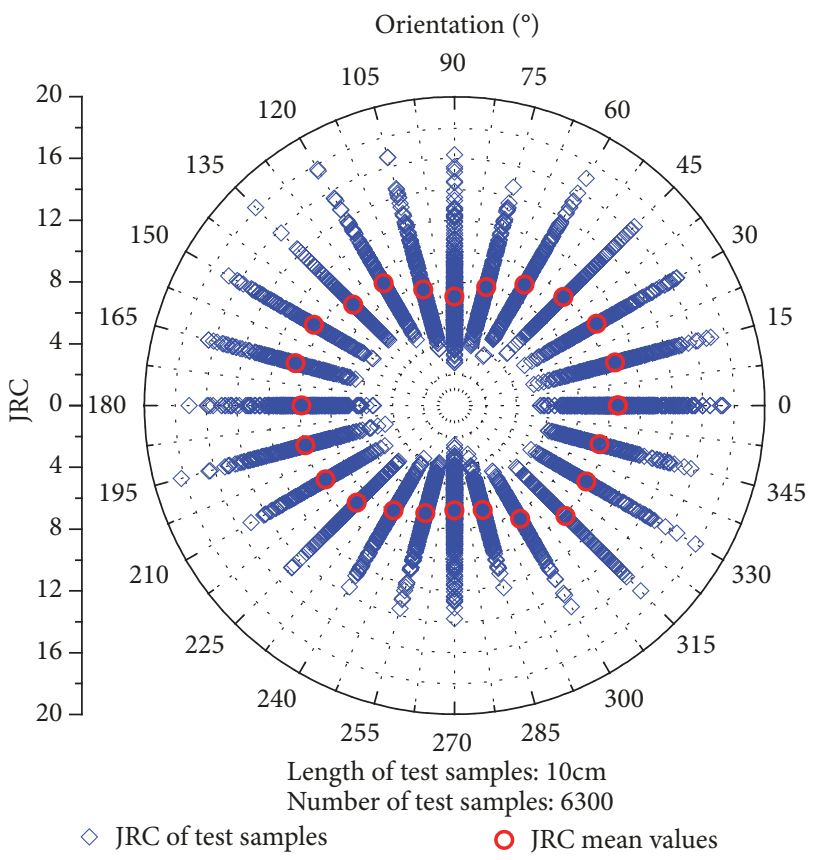

FIGURE 3: Polar plots of directional $J R C$ values of $10 \mathrm{~cm}$ joint samples on the slate fracture surface.

$73.37 \%, 72.41 \%$, and $71.19 \%$ of the directional joint roughness samples fell within the range $\left[J R C_{\text {mean }}-\sigma, J R C_{\text {mean }}+\sigma\right]$. The polar plots of the upper and lower limits of $J R C$ values showed a pattern similar to an ellipse (Figure 5). The parametric equations for each anisotropic ellipse are

$$
\begin{aligned}
& x=a \cos \theta \\
& y=b \sin \theta,
\end{aligned}
$$

where $x, y$ are the coordinates of any point on the ellipse; $a$ and $b$ are the radius on the $x$ and $y$ axes, respectively, and $\theta$ is the orientation, which ranges from 0 to $2 \pi$ in radians.

For joint samples of each size, two ellipse envelopes can be established to fit the upper and lower limits of $J R C$ values based on the least square method (LSM). The JRC values indicating the anisotropy characteristics should be spread among the two ellipse envelopes. According to the concept of NNs, the parametric equations with NNs for JRC are

$$
\begin{aligned}
& x=\left(n_{1}+\mu_{n 1} I\right) \cos \theta \\
& y=\left(n_{2}+\mu_{n 2} I\right) \sin \theta, \\
& I \in[0,0.5],
\end{aligned}
$$

where $n_{1}, n_{2}$ are the radius of the inside ellipse envelope; $\mu_{n 1}$, $\mu_{n 2}$ are twice the difference in radius of the outside and inside ellipse envelopes. For example, $n_{1}, n_{2}$ of the inside ellipse envelope were 8.655 and 5.808 , and $n_{3}, n_{4}$ of the outside ellipse envelope were 13.074 and 9.924 (Figure 5). Then, the $\mu_{n 1}, \mu_{n 2}$ terms can be calculated as follows:

$$
\begin{aligned}
& \mu_{n 1}=2 \times(13.074-8.655)=8.838 \\
& \mu_{n 2}=2 \times(9.924-5.808)=8.232
\end{aligned}
$$




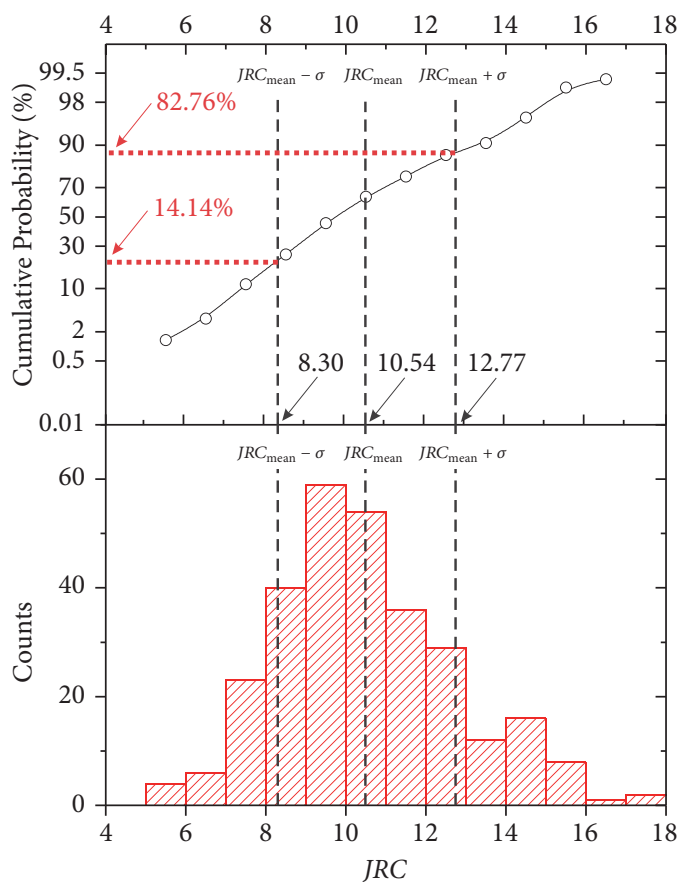

(a)

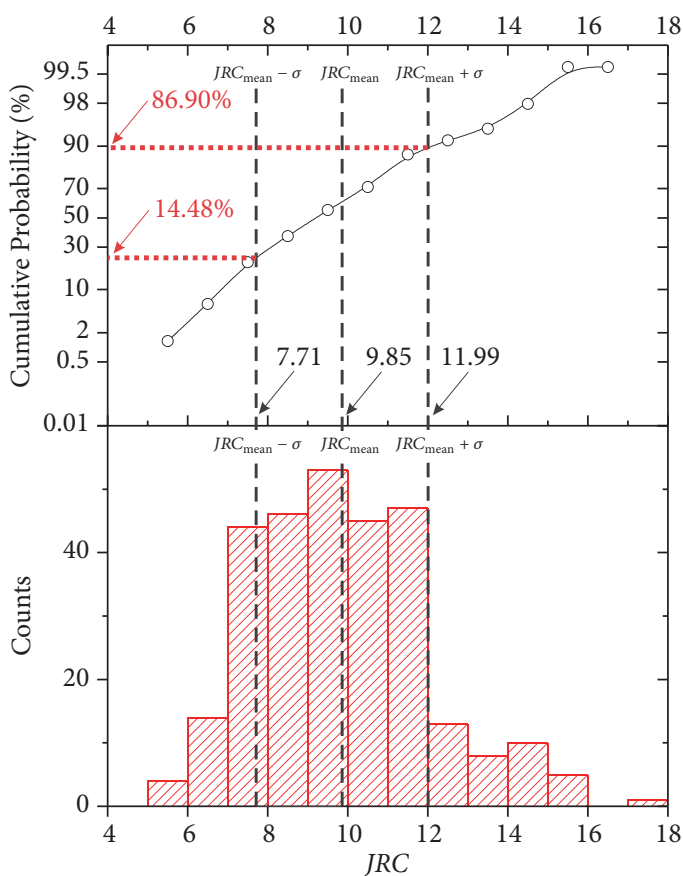

(c)

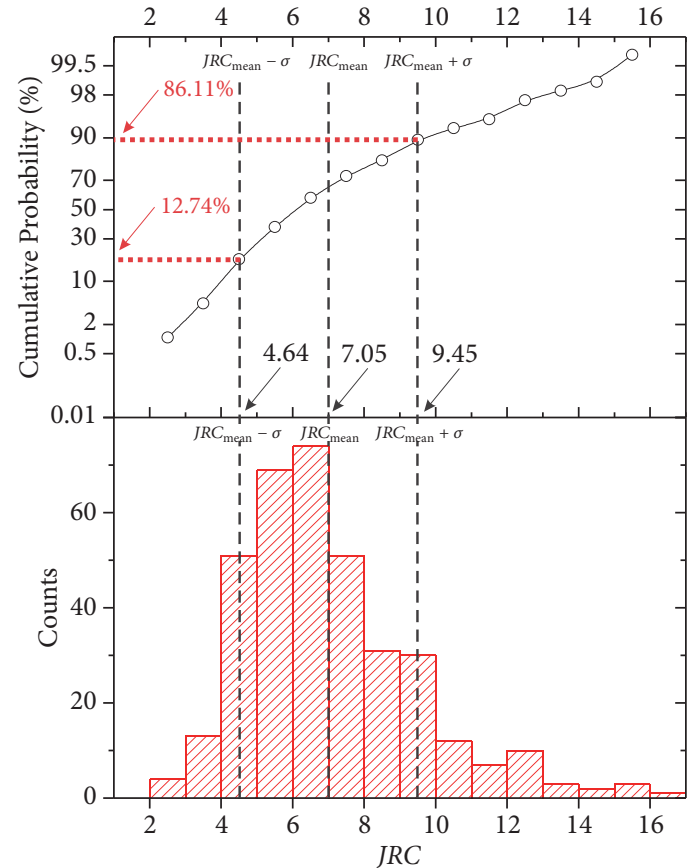

(b)

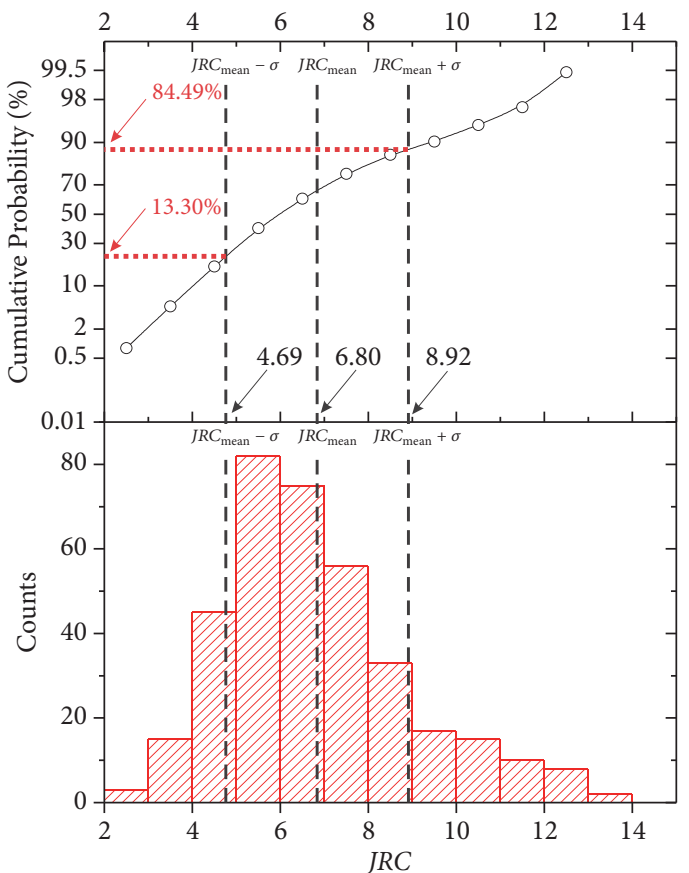

(d)

Figure 4: Frequency distributions of $10 \mathrm{~cm}$ joint samples in the orientations of (a) $0^{\circ}$, (b) $90^{\circ}$, (c) $180^{\circ}$, and (d) $270^{\circ}$.

Thus, the neutrosophic functions based on the parametric equations with NNs for describing the anisotropy characteristic of $10 \mathrm{~cm}$ joint samples were

$$
\begin{aligned}
& x=(8.655+8.838 \cdot I) \cos \theta \\
& y=(5.808+8.232 \cdot I) \sin \theta, \\
& I \in[0,0.5] .
\end{aligned}
$$

The fitting results of two NN radiuses $\left(a=n_{1}+\mu_{n 1} I\right.$ and $b=n_{2}+\mu_{n 2} I$ for $\left.I \in[0,0.5]\right)$ using the neutrosophic functions based on the parametric equations for the anisotropy characteristics of different sized joint samples are tabulated in Table 1.

By using (3) and (10), the directional JRC values with NN functions, $J R C(\theta)$, within $I \in[0,0.5]$ can be derived by 


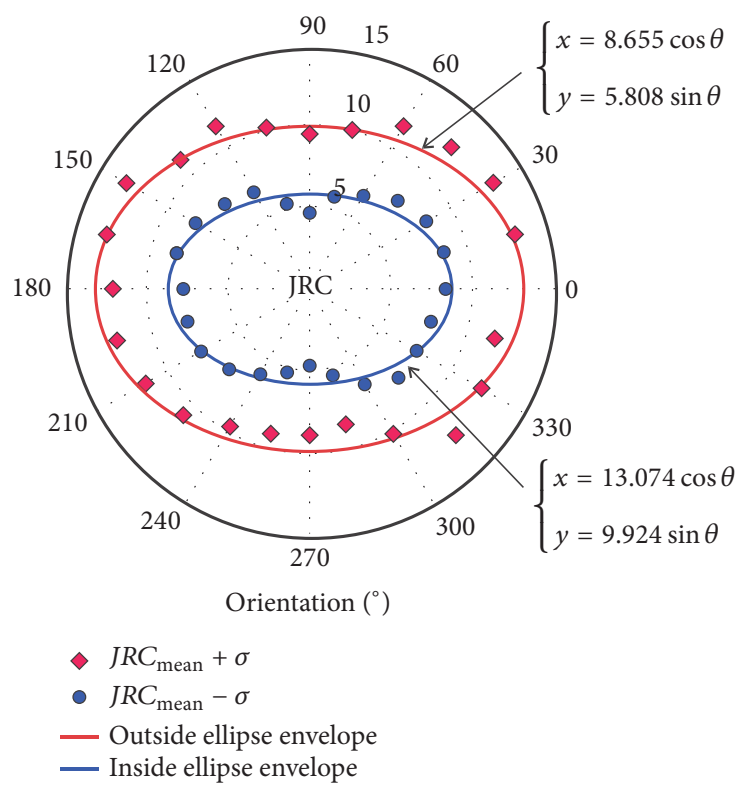

FigURE 5: Upper and lower limits of $J R C$ values and the corresponding ellipse fitting envelopes.

TABLE 1: Fitting results of NNs for the $J R C$ values of different sized joint samples.

\begin{tabular}{lcccc}
\hline Scale $(\mathrm{cm})$ & $n_{1}$ & $\mu_{n 1}$ & $n_{2}$ & $\mu_{n 2}$ \\
\hline 10 & 8.655 & 8.838 & 5.808 & 8.232 \\
20 & 8.332 & 7.009 & 5.710 & 6.467 \\
30 & 8.028 & 6.455 & 5.548 & 5.477 \\
40 & 7.902 & 5.978 & 5.479 & 4.668 \\
50 & 7.638 & 5.925 & 5.343 & 4.349 \\
60 & 7.476 & 5.639 & 5.524 & 4.976 \\
70 & 7.503 & 5.229 & 5.246 & 4.602 \\
80 & 7.401 & 4.693 & 5.137 & 4.179 \\
90 & 7.271 & 4.256 & 5.097 & 3.735 \\
100 & 7.178 & 3.946 & 5.045 & 3.319 \\
\hline
\end{tabular}

$$
\begin{aligned}
J R C(\theta) & =\sqrt{x^{2}+y^{2}}=\sqrt{\left(\left(n_{1}+\mu_{n 1} I\right) \cos \theta\right)^{2}+\left(\left(n_{2}+\mu_{n 2} I\right) \sin \theta\right)^{2}} \\
& =\sqrt{\left(n_{1}+\mu_{n 1} I\right) \cdot\left(n_{1}+\mu_{n 1} I\right) \cos ^{2} \theta+\left(n_{2}+\mu_{n 2} I\right) \cdot\left(n_{2}+\mu_{n 2} I\right) \sin ^{2} \theta} \\
& =\sqrt{\left[n_{1}^{2},\left(n_{1}+\frac{\mu_{n 1}}{2}\right)^{2}\right] \cos ^{2} \theta+\left[n_{2}^{2},\left(n_{2}+\frac{\mu_{n 2}}{2}\right)^{2}\right] \sin ^{2} \theta} \\
& =\left[\sqrt{n_{1}{ }^{2} \cos ^{2} \theta+n_{2}{ }^{2} \sin ^{2} \theta}, \sqrt{\left(n_{1}+\frac{\mu_{n 1}}{2}\right)^{2} \cos ^{2} \theta+\left(n_{2}+\frac{\mu_{n 2}}{2}\right)^{2} \sin ^{2} \theta}\right]
\end{aligned}
$$

According to the fitting results of NNs of different sized joint samples in Table 1, the JRC neutrosophic functions of different sized joint profiles in each orientation can be obtained by using (13). The calculated results are tabulated in Table 2.

\section{Neutrosophic Function with NNs for Scale Effect of Joint Surface Roughness}

The mean $J R C$ values in various orientations were tabulated (Table 3). For each orientation, as the mean JRC value for 
TABLE 2: Neutrosophic function values of $\operatorname{JRC}(\theta)$ within $I \in[0,0.5]$ (indeterminate intervals) for different sized joint samples in various orientations.

\begin{tabular}{|c|c|c|c|c|c|}
\hline \multirow{2}{*}{$\begin{array}{l}\text { Orientation } \\
\left({ }^{\circ}\right)\end{array}$} & \multicolumn{5}{|c|}{$J R C$} \\
\hline & $10 \mathrm{~cm}$ & $20 \mathrm{~cm}$ & $30 \mathrm{~cm}$ & $40 \mathrm{~cm}$ & $50 \mathrm{~cm}$ \\
\hline 0 & {$[8.655,13.074]$} & {$[8.332,11.837]$} & {$[8.028,11.255]$} & {$[7.902,10.891]$} & {$[7.638,10.600]$} \\
\hline 15 & {$[8.494,12.887]$} & {$[8.183,11.665]$} & {$[7.886,11.081]$} & {$[7.763,10.712]$} & {$[7.506,10.422]$} \\
\hline 30 & {$[8.038,12.362]$} & {$[7.760,11.184]$} & {$[7.485,10.591]$} & {$[7.371,10.209]$} & {$[7.134,9.920]$} \\
\hline 45 & {$[7.370,11.607]$} & {$[7.143,10.491]$} & {$[6.900,9.883]$} & {$[6.799,9.478]$} & {$[6.591,9.189]$} \\
\hline 60 & {$[6.635,10.798]$} & {$[6.466,9.748]$} & {$[6.261,9.120]$} & {$[6.174,8.685]$} & {$[6.000,8.395]$} \\
\hline 75 & {$[6.041,10.165]$} & {$[5.922,9.166]$} & {$[5.748,8.518]$} & {$[5.674,8.056]$} & {$[5.527,7.763]$} \\
\hline 90 & {$[5.808,9.924]$} & {$[5.710,8.944]$} & {$[5.548,8.287]$} & {$[5.479,7.813]$} & {$[5.343,7.518]$} \\
\hline 105 & {$[6.041,10.165]$} & {$[5.922,9.166]$} & {$[5.748,8.518]$} & {$[5.674,8.056]$} & {$[5.527,7.763]$} \\
\hline 120 & {$[6.635,10.798]$} & {$[6.466,9.748]$} & {$[6.261,9.120]$} & {$[6.174,8.685]$} & {$[6.000,8.395]$} \\
\hline 135 & {$[7.370,11.607]$} & {$[7.143,10.491]$} & {$[6.900,9.883]$} & {$[6.799,9.478]$} & {$[6.591,9.189]$} \\
\hline 150 & {$[8.038,12.362]$} & {$[7.760,11.184]$} & {$[7.485,10.591]$} & {$[7.371,10.209]$} & {$[7.134,9.920]$} \\
\hline 165 & {$[8.494,12.887]$} & {$[8.183,11.665]$} & {$[7.886,11.081]$} & {$[7.763,10.712]$} & {$[7.506,10.422]$} \\
\hline 180 & {$[8.655,13.074]$} & {$[8.332,11.837]$} & {$[8.028,11.255]$} & {$[7.902,10.891]$} & {$[7.638,10.600]$} \\
\hline 195 & {$[8.494,12.887]$} & {$[8.183,11.665]$} & {$[7.886,11.081]$} & {$[7.763,10.712]$} & {$[7.506,10.422]$} \\
\hline 210 & {$[8.038,12.362]$} & {$[7.760,11.184]$} & {$[7.485,10.591]$} & {$[7.371,10.209]$} & {$[7.134,9.920]$} \\
\hline 225 & {$[7.370,11.607]$} & {$[7.143,10.491]$} & {$[6.900,9.883]$} & {$[6.799,9.478]$} & {$[6.591,9.189]$} \\
\hline 240 & {$[6.635,10.798]$} & {$[6.466,9.748]$} & {$[6.261,9.120]$} & {$[6.174,8.685]$} & {$[6.000,8.395]$} \\
\hline 255 & {$[6.041,10.165]$} & {$[5.922,9.166]$} & {$[5.748,8.518]$} & {$[5.674,8.056]$} & {$[5.527,7.763]$} \\
\hline 270 & {$[5.808,9.924]$} & {$[5.710,8.944]$} & {$[5.548,8.287]$} & {$[5.479,7.813]$} & {$[5.343,7.518]$} \\
\hline 285 & {$[6.041,10.165]$} & {$[5.922,9.166]$} & {$[5.748,8.518]$} & {$[5.674,8.056]$} & {$[5.527,7.763]$} \\
\hline 300 & {$[6.635,10.798]$} & {$[6.466,9.748]$} & {$[6.261,9.120]$} & {$[6.174,8.685]$} & {$[6.000,8.395]$} \\
\hline 315 & {$[7.370,11.607]$} & {$[7.143,10.491]$} & {$[6.900,9.883]$} & {$[6.799,9.478]$} & {$[6.591,9.189]$} \\
\hline 330 & {$[8.038,12.362]$} & {$[7.760,11.184]$} & {$[7.485,10.591]$} & {$[7.371,10.209]$} & {$[7.134,9.920]$} \\
\hline 345 & {$[8.494,12.887]$} & {$[8.183,11.665]$} & {$[7.886,11.081]$} & {$[7.763,10.712]$} & {$[7.506,10.422]$} \\
\hline 360 & {$[8.655,13.074]$} & {$[8.332,11.837]$} & {$[8.028,11.255]$} & {$[7.902,10.891]$} & {$[7.638,10.600]$} \\
\hline Orientation & & & $J R C$ & & \\
\hline$\left({ }^{\circ}\right)$ & $60 \mathrm{~cm}$ & $70 \mathrm{~cm}$ & $80 \mathrm{~cm}$ & $90 \mathrm{~cm}$ & $100 \mathrm{~cm}$ \\
\hline 0 & {$[7.476,10.295]$} & {$[7.503,10.117]$} & {$[7.401,9.747]$} & {$[7.271,9.399]$} & {$[7.178,9.151]$} \\
\hline 15 & {$[7.361,10.158]$} & {$[7.373,9.966]$} & {$[7.271,9.599]$} & {$[7.146,9.256]$} & {$[7.055,9.008]$} \\
\hline 30 & {$[7.039,9.774]$} & {$[7.007,9.540]$} & {$[6.905,9.182]$} & {$[6.793,8.853]$} & {$[6.708,8.605]$} \\
\hline 45 & {$[6.573,9.224]$} & {$[6.473,8.925]$} & {$[6.370,8.580]$} & {$[6.279,8.272]$} & {$[6.204,8.021]$} \\
\hline 60 & {$[6.071,8.639]$} & {$[5.892,8.265]$} & {$[5.787,7.932]$} & {$[5.718,7.646]$} & {$[5.654,7.392]$} \\
\hline 75 & {$[5.676,8.185]$} & {$[5.426,7.746]$} & {$[5.319,7.422]$} & {$[5.270,7.153]$} & {$[5.215,6.895]$} \\
\hline 90 & {$[5.524,8.012]$} & {$[5.246,7.547]$} & {$[5.137,7.227]$} & {$[5.097,6.964]$} & {$[5.045,6.704]$} \\
\hline 105 & {$[5.676,8.185]$} & {$[5.426,7.746]$} & {$[5.319,7.422]$} & {$[5.270,7.153]$} & {$[5.215,6.895]$} \\
\hline 120 & {$[6.071,8.639]$} & {$[5.892,8.265]$} & {$[5.787,7.932]$} & {$[5.718,7.646]$} & {$[5.654,7.392]$} \\
\hline 135 & {$[6.573,9.224]$} & {$[6.473,8.925]$} & {$[6.370,8.580]$} & {$[6.279,8.272]$} & {$[6.204,8.021]$} \\
\hline 150 & {$[7.039,9.774]$} & {$[7.007,9.540]$} & {$[6.905,9.182]$} & {$[6.793,8.853]$} & {$[6.708,8.605]$} \\
\hline 165 & {$[7.361,10.158]$} & {$[7.373,9.966]$} & {$[7.271,9.599]$} & {$[7.146,9.256]$} & {$[7.055,9.008]$} \\
\hline 180 & {$[7.476,10.295]$} & {$[7.503,10.117]$} & {$[7.401,9.747]$} & {$[7.271,9.399]$} & {$[7.178,9.151]$} \\
\hline 195 & {$[7.361,10.158]$} & {$[7.373,9.966]$} & {$[7.271,9.599]$} & {$[7.146,9.256]$} & {$[7.055,9.008]$} \\
\hline 210 & {$[7.039,9.774]$} & {$[7.007,9.540]$} & {$[6.905,9.182]$} & {$[6.793,8.853]$} & {$[6.708,8.605]$} \\
\hline 225 & {$[6.573,9.224]$} & {$[6.473,8.925]$} & {$[6.370,8.580]$} & {$[6.279,8.272]$} & {$[6.204,8.021]$} \\
\hline 240 & {$[6.071,8.639]$} & {$[5.892,8.265]$} & {$[5.787,7.932]$} & {$[5.718,7.646]$} & {$[5.654,7.392]$} \\
\hline 255 & {$[5.676,8.185]$} & {$[5.426,7.746]$} & {$[5.319,7.422]$} & {$[5.270,7.153]$} & {$[5.215,6.895]$} \\
\hline 270 & {$[5.524,8.012]$} & {$[5.246,7.547]$} & {$[5.137,7.227]$} & {$[5.097,6.964]$} & {$[5.045,6.704]$} \\
\hline 285 & {$[5.676,8.185]$} & {$[5.426,7.746]$} & {$[5.319,7.422]$} & {$[5.270,7.153]$} & {$[5.215,6.895]$} \\
\hline 300 & {$[6.071,8.639]$} & {$[5.892,8.265]$} & {$[5.787,7.932]$} & {$[5.718,7.646]$} & {$[5.654,7.392]$} \\
\hline 315 & {$[6.573,9.224]$} & {$[6.473,8.925]$} & {$[6.370,8.580]$} & {$[6.279,8.272]$} & {$[6.204,8.021]$} \\
\hline 330 & {$[7.039,9.774]$} & {$[7.007,9.540]$} & {$[6.905,9.182]$} & {$[6.793,8.853]$} & {$[6.708,8.605]$} \\
\hline 345 & {$[7.361,10.158]$} & {$[7.373,9.966]$} & {$[7.271,9.599]$} & {$[7.146,9.256]$} & {$[7.055,9.008]$} \\
\hline 360 & {$[7.476,10.295]$} & {$[7.503,10.117]$} & {$[7.401,9.747]$} & {$[7.271,9.399]$} & {$[7.178,9.151]$} \\
\hline
\end{tabular}


TABLE 3: Summary of mean JRC values obtained in different orientations and their variations with scale.

\begin{tabular}{|c|c|c|c|c|c|c|c|c|c|c|}
\hline \multirow{2}{*}{$\begin{array}{l}\text { Orientations } \\
\left({ }^{\circ}\right)\end{array}$} & \multicolumn{10}{|c|}{ Mean $J R C$ values } \\
\hline & $J R C_{10}$ & $J R C_{20}$ & $J R C_{30}$ & $J R C_{40}$ & $J R C_{50}$ & $J R C_{60}$ & $J R C_{70}$ & $J R C_{80}$ & $J R C_{90}$ & $J R C_{100}$ \\
\hline 0 & 10.536 & 9.653 & 9.273 & 8.975 & 8.822 & 8.802 & 8.682 & 8.601 & 8.568 & 8.463 \\
\hline 15 & 10.711 & 9.968 & 9.343 & 9.271 & 9.230 & 8.973 & 8.833 & 8.587 & 8.360 & 8.140 \\
\hline 30 & 10.594 & 9.929 & 9.572 & 9.121 & 9.092 & 8.601 & 8.760 & 8.571 & 8.293 & 8.104 \\
\hline 45 & 9.924 & 9.201 & 9.008 & 8.508 & 8.334 & 8.624 & 8.326 & 8.077 & 7.846 & 7.573 \\
\hline 60 & 9.025 & 8.405 & 7.884 & 7.794 & 7.187 & 8.268 & 7.360 & 7.138 & 6.872 & 6.713 \\
\hline 75 & 7.935 & 7.460 & 6.773 & 6.306 & 6.545 & 6.499 & 6.244 & 6.093 & 5.950 & 5.832 \\
\hline 90 & 7.047 & 6.691 & 6.338 & 5.999 & 6.148 & 6.089 & 5.954 & 5.893 & 5.826 & 5.822 \\
\hline 105 & 7.777 & 7.222 & 6.677 & 6.232 & 6.463 & 6.461 & 6.167 & 5.992 & 5.890 & 5.836 \\
\hline 120 & 9.132 & 8.521 & 8.200 & 7.967 & 7.321 & 7.824 & 7.247 & 7.065 & 6.854 & 6.691 \\
\hline 135 & 9.226 & 8.567 & 8.090 & 7.819 & 7.374 & 7.666 & 7.385 & 7.087 & 6.915 & 6.759 \\
\hline 150 & 10.467 & 9.565 & 8.910 & 8.906 & 8.393 & 8.816 & 8.206 & 8.015 & 7.656 & 7.444 \\
\hline 165 & 10.604 & 9.965 & 9.532 & 8.876 & 8.612 & 8.646 & 8.393 & 8.111 & 7.905 & 7.717 \\
\hline 180 & 9.850 & 9.098 & 8.757 & 8.600 & 8.297 & 8.127 & 7.965 & 7.898 & 7.834 & 7.829 \\
\hline 195 & 9.938 & 9.230 & 8.678 & 8.799 & 8.531 & 8.102 & 7.922 & 7.656 & 7.461 & 7.313 \\
\hline 210 & 9.590 & 8.941 & 8.653 & 8.260 & 8.207 & 7.383 & 7.753 & 7.505 & 7.249 & 7.048 \\
\hline 225 & 8.917 & 8.255 & 8.133 & 7.701 & 7.680 & 7.437 & 7.318 & 7.131 & 6.865 & 6.674 \\
\hline 240 & 7.858 & 7.303 & 6.824 & 6.743 & 6.325 & 6.818 & 6.353 & 6.152 & 5.914 & 6.685 \\
\hline 255 & 7.217 & 6.864 & 6.335 & 6.105 & 6.033 & 6.069 & 5.892 & 5.712 & 5.780 & 5.395 \\
\hline 270 & 6.802 & 6.312 & 6.006 & 5.882 & 5.787 & 5.971 & 5.853 & 5.738 & 5.826 & 5.586 \\
\hline 285 & 7.006 & 6.494 & 6.111 & 5.845 & 5.756 & 6.061 & 5.840 & 5.639 & 5.472 & 5.363 \\
\hline 300 & 8.472 & 7.812 & 7.530 & 7.281 & 6.953 & 7.067 & 6.800 & 6.641 & 6.446 & 6.310 \\
\hline 315 & 10.143 & 9.455 & 8.964 & 8.570 & 8.122 & 8.686 & 8.366 & 7.658 & 7.464 & 7.354 \\
\hline 330 & 9.829 & 9.001 & 8.326 & 8.329 & 7.871 & 8.053 & 7.913 & 7.650 & 7.347 & 7.093 \\
\hline 345 & 9.683 & 9.176 & 8.773 & 8.474 & 7.860 & 7.848 & 7.727 & 7.467 & 7.178 & 7.004 \\
\hline 360 & 10.536 & 9.653 & 9.273 & 8.975 & 8.822 & 8.802 & 8.682 & 8.601 & 8.568 & 8.463 \\
\hline
\end{tabular}

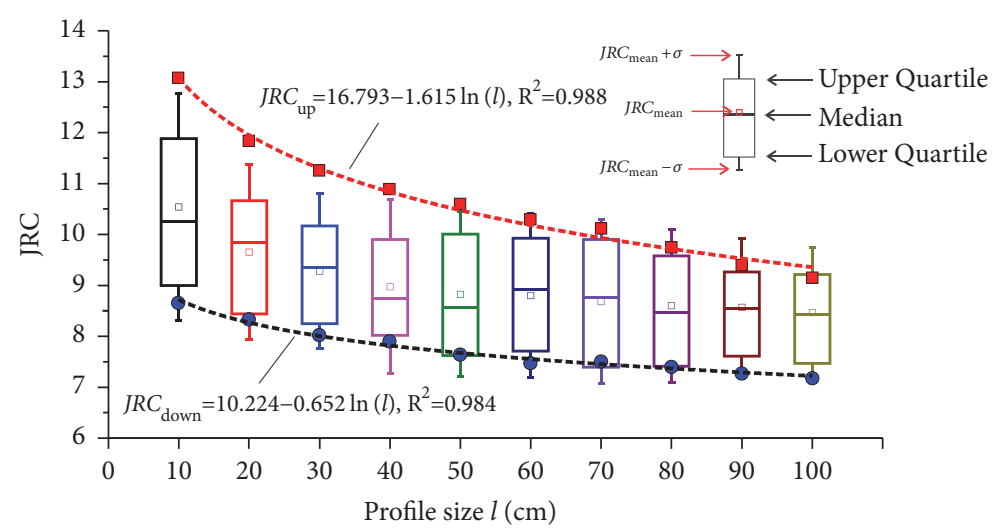

FIGURE 6: JRC values of different sized joint samples in the orientation of $0^{\circ}$.

each sample size decreased, it showed a negative scale effect. For example, the $J R C$ values of different sized joint samples in the orientation of $0^{\circ}$ were illustrated in Figure 6 , according to the calculated $J R C$ intervals. The upper and lower limits of $J R C$ values were shown in Figure 6. Both the upper and lower limits decreased as the joint sample length increased. These decreasing trends are fitted by logarithmic functions:

$$
\begin{aligned}
J R C_{\text {down }} & =m_{1}+m_{2} \cdot \ln (l) \\
J R C_{\text {up }} & =m_{3}+m_{4} \cdot \ln (l)
\end{aligned}
$$

where $J R C_{\text {down }}$ and $J R C_{\text {up }}$ are the fitting values of the joint roughness coefficient of different sized test profile; $m_{1}, m_{2}$, $m_{3}$, and $m_{4}$ are fitting parameters of the logarithmic functions revealing the indeterminacy.

According to the concept of NNs, the neutrosophic function $\operatorname{JRC}(l)$ for $I \in[0,0.5]$ is written as

$$
\begin{aligned}
& J R C(l)=\left(m_{1}+\mu_{m_{1}} I\right)+\left(m_{2}+\mu_{m_{2}} I\right) * \ln (l)=\left(m_{1}\right. \\
& \left.+m_{2} \ln (l)\right)+\left(\mu_{m_{1}}+\mu_{m_{2}} \ln (l)\right) I=\left[m_{1}\right.
\end{aligned}
$$




$$
\begin{array}{r}
\left.+m_{2} \ln (l), m_{1}+m_{2} \ln (l)+\frac{\left(\mu_{m_{1}}+\mu_{m_{2}} \ln (l)\right)}{2}\right], \\
I \in[0,0.5] .
\end{array}
$$

where $m_{1}, m_{2}$ are the fitting parameters for the logarithmic function of the lower bound values and $\mu_{m 1}, \mu_{m 2}$ are taken as twice the difference in the fitting parameters for the logarithmic function between the lower and upper bound values. For example, $m_{1}, m_{2}$ of different sized joint samples in the orientation of $0^{\circ}$ were 10.224 and -0.652 , and $m_{3}, m_{4}$ of the outside ellipse envelope were 16.793 and -1.615 (Figure 5). Then,

$$
\begin{aligned}
& \mu_{m 1}=2 \times(16.793-10.224)=13.138 \\
& \mu_{m 2}=2 \times(-1.615+0.652)=-1.926
\end{aligned}
$$

Thus, the NN function $J R C(l)$ with $I \in[0,0.5]$ for describing the $J R C$ values of different sized joint samples in the orientation of $0^{\circ}$ was

$$
\begin{aligned}
J R C(l)= & (10.224+13.138 I)+(-0.652-1.926 I) \\
& * \ln (l) \\
= & {[10.224-0.652 \ln (l), 16.793-1.615 \ln (l)] }
\end{aligned}
$$

$$
\begin{aligned}
\psi & =\frac{a}{b}=\frac{n_{1}+\mu_{n_{1}} I}{n_{2}+\mu_{n_{2}} I}, \quad I \in[0,0.5] . \\
= & {\left[\begin{array}{l}
\min \left(\frac{n_{1}+\mu_{n_{1}} \times 0}{n_{2}+\mu_{n_{2}} \times 0.5}, \frac{n_{1}+\mu_{n_{1}} \times 0}{n_{2}+\mu_{n_{2}} \times 0}, \frac{n_{1}+\mu_{n_{1}} \times 0.5}{n_{2}+\mu_{n_{2}} \times 0.5}, \frac{n_{1}+\mu_{n_{1}} \times 0.5}{n_{2}+\mu_{n_{2}} \times 0}\right), \\
\max \left(\frac{n_{1}+\mu_{n_{1}} \times 0}{n_{2}+\mu_{n_{2}} \times 0.5}, \frac{n_{1}+\mu_{n_{1}} \times 0}{n_{2}+\mu_{n_{2}} \times 0}, \frac{n_{1}+\mu_{n_{1}} \times 0.5}{n_{2}+\mu_{n_{2}} \times 0.5}, \frac{n_{1}+\mu_{n_{1}} \times 0.5}{n_{2}+\mu_{n_{2}} \times 0}\right)
\end{array}\right]=\left[\frac{2 n_{1}}{2 n_{2}+\mu_{n_{2}}}, \frac{2 n_{1}+\mu_{n_{1}}}{2 n_{2}}\right] }
\end{aligned}
$$

The fitting results of two NNs $\left(z=m_{1}+\mu_{m 1} I\right.$ and $z=m_{2}+\mu_{m 2} I$ for $\left.I \in[0,0.5]\right)$ in the neutrosophic function $J R C(l)$ for anisotropy characteristics of different sized joint samples were tabulated in Table 4.

\section{Neutrosophic Parameter for Evaluating the Roughness Anisotropy}

Tatone [28] quantified the roughness anisotropy using the according to the polar plots of the roughness parameters. The parameter $\psi=a / b$, i.e., the ratio of major and minor principal axis, was utilized to indicate the anisotropic degree. However, both $a$ and $b$ are NNs, which consist of determinate and indeterminate parts, as shown below:

$$
\begin{aligned}
& a=n_{1}+\mu_{n 1} I \\
& b=n_{2}+\mu_{n 2} I, \\
& I \in[0,0.5] .
\end{aligned}
$$

Using (4), a neutrosophic parameter $\psi$ for evaluating the roughness anisotropy can be obtained as follows: ratio of the maximum and minimum directional roughness
Figure 7 illustrates the upper and lower bounds of the parameter $\psi$ for different sized joint samples. The difference between the $\psi_{\text {up }}$ and $\psi_{\text {down }}$ decreased with the sample size and approached a certain value when the sample size exceeded 60 $\mathrm{cm}$.

\section{Conclusion}

The statistical assessment of $J R C$ values was applied to analyze the roughness characteristics considering both the sample size and the measurement orientation. Basic operations of NNs were first introduced. The neutrosophic functions $J R C(\theta)$ and $J R C(l)$ were developed for analyzing and expressing the anisotropy characteristics and scale effect of joint surface roughness. The neutrosophic function $\operatorname{JRC}(\theta)$ for anisotropic ellipse was derived according to the statistical assessment data of $J R C$ of joint profiles in various orientations. The upper and lower limits of $J R C$ values decreased with the increasing sampling length, showing a negative scale effect on joint roughness. The neutrosophic function $J R C(l)$ was then acquired to determine the $J R C$ values of different sized joint samples. The neutrosophic parameter $\psi$ was developed according to the ratio of maximum and minimum directional roughness. This was proved to be a new parameter for indicating the relationship between the scale effect and the anisotropy of joint roughness. The neutrosophic functions efficiently and conveniently showed both the anisotropy characteristic and the scale effect of the surface roughness of rock joints.

\section{Data Availability}

The data used to support the findings of this study are included within the article. 
TABLE 4: Fitting results of NNs in $J R C(l)$ for $I \in[0,0.5]$ corresponding to the $J R C$ values of joint samples in different orientations.

\begin{tabular}{lcccc}
\hline Orientation $\left(^{\circ}\right)$ & $m_{1}$ & $\mu_{\mathrm{m} 1}$ & $m_{2}$ & $\mu_{\mathrm{m} 2}$ \\
\hline 0 & 10.224 & 13.138 & -0.652 & -1.926 \\
15 & 10.025 & 13.057 & -0.635 & -1.918 \\
30 & 9.459 & 12.850 & -0.586 & -1.902 \\
45 & 8.629 & 12.586 & -0.514 & -1.886 \\
60 & 7.710 & 12.360 & -0.431 & -1.880 \\
75 & 6.964 & 12.239 & -0.362 & -1.885 \\
90 & 6.669 & 12.215 & -0.334 & -1.892 \\
105 & 6.964 & 12.239 & -0.362 & -1.885 \\
120 & 7.710 & 12.360 & -0.431 & -1.880 \\
135 & 8.629 & 12.586 & -0.514 & -1.886 \\
150 & 9.459 & 12.850 & -0.586 & -1.902 \\
165 & 10.025 & 13.057 & -0.635 & -1.918 \\
180 & 10.224 & 13.138 & -0.652 & -1.925 \\
195 & 10.025 & 13.057 & -0.635 & -1.918 \\
210 & 9.459 & 12.850 & -0.586 & -1.902 \\
225 & 8.629 & 12.586 & -0.514 & -1.886 \\
240 & 7.710 & 12.360 & -0.431 & -1.880 \\
255 & 6.964 & 12.239 & -0.362 & -1.885 \\
270 & 6.669 & 12.215 & -0.334 & -1.892 \\
285 & 6.964 & 12.239 & -0.362 & -1.885 \\
300 & 7.710 & 12.360 & -0.431 & -1.880 \\
315 & 8.629 & 12.586 & -0.514 & -1.886 \\
330 & 9.459 & 12.850 & -0.586 & -1.902 \\
345 & 10.025 & 13.057 & -0.635 & -1.918 \\
360 & 10.224 & 13.138 & -0.652 & -1.926 \\
\hline & & & &
\end{tabular}

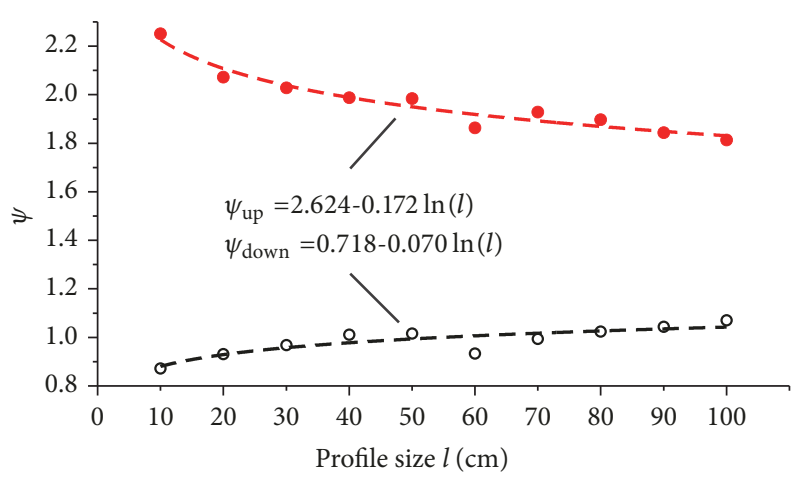

FIGURE 7: Upper and lower bounds of the parameter $\psi$ of different sized joint samples.

\section{Conflicts of Interest}

The authors declare that they have no conflicts of interest.

\section{Acknowledgments}

The study was funded by National Natural Science Foundation of China (nos. 41502300, 41427802, 41572299), Zhejiang Provincial Natural Science Foundation (no. LQ16D020001).

\section{References}

[1] A. Ghazvinian, M. J. Azinfar, and P. Norozi, "Mechanical response of discontinuities of different joint wall contact strengths," Arabian Journal of Geosciences, vol. 6, no. 11, pp. 4419-4426, 2013.

[2] M. Sanei, L. Faramarzi, S. Goli, A. Fahimifar, A. Rahmati, and A. Mehinrad, "Development of a new equation for joint roughness coefficient (JRC) with fractal dimension: a case study of Bakhtiary Dam site in Iran," Arabian Journal of Geosciences, vol. 8, no. 1, pp. 465-475, 2015.

[3] R.-H. Cao, P. Cao, H. Lin, G. Ma, and Y. Chen, "Failure characteristics of intermittent fissures under a compressiveshear test: experimental and numerical analyses," Theoretical and Applied Fracture Mechanics, vol. 96, pp. 740-757, 2018.

[4] H. Lin, H. Wang, X. Fan, P. Cao, and K. Zhou, "Particle size distribution effects on deformation properties of graded aggregate base under cyclic loading," European Journal of Environmental and Civil Engineering, pp. 1-18, 2017.

[5] R. Yong, J. Ye, Q.-F. Liang, M. Huang, and S.-G. Du, "Estimation of the joint roughness coefficient (JRC) of rock joints by vector similarity measures," Bulletin of Engineering Geology and the Environment, vol. 77, no. 2, pp. 735-749, 2018.

[6] N. Barton, "Review of a new shear-strength criterion for rock joints," Engineering Geology, vol. 7, no. 4, pp. 287-332, 1973.

[7] R. Tse and D. Cruden, "Estimating joint roughness coefficients," International Journal of Rock Mechanics and Mining Sciences \& Geomechanics Abstracts, vol. 16, no. 5, pp. 303-307, 1979.

[8] Y.-H. Lee, J. R. Carr, D. J. Barr, and C. J. Haas, "The fractal dimension as a measure of the roughness of rock discontinuity profiles," International Journal of Rock Mechanics and Mining Sciences \& Geomechanics Abstracts, vol. 27, no. 6, pp. 453-464, 1990.

[9] N. Maerz, J. Franklin, and C. Bennett, "Joint roughness measurement using shadow profilometry," International Journal of Rock Mechanics and Mining Sciences \& Geomechanics Abstracts, vol. 27, no. 5, pp. 329-343, 1990.

[10] G. Grasselli, J. Wirth, and P. Egger, "Quantitative threedimensional description of a rough surface and parameter evolution with shearing," International Journal of Rock Mechanics and Mining Sciences, vol. 39, no. 6, pp. 789-800, 2002.

[11] H.-S. Jang, S.-S. Kang, and B.-A. Jang, "Determination of joint roughness coefficients using roughness parameters," Rock Mechanics and Rock Engineering, vol. 47, no. 6, pp. 2061-2073, 2014.

[12] P. Alameda-Hernández, J. Jiménez-Perálvarez, J. A. Palenzuela et al., "Improvement of the JRC calculation using different parameters obtained through a new survey method applied to rock discontinuities," Rock Mechanics and Rock Engineering, vol. 47, no. 6, pp. 2047-2060, 2014.

[13] S. G. Du, M. Huang, Z. Y. Luo, R. D. Jia, and Y. M. Wang, "Scale effect of undulation amplitude of rock joints," Engineering Geology, vol. 18, pp. 47-52, 2010.

[14] S. J. Chen, W. C. Zhu, S. X. Liu, F. Zhang, and L. F. Guo, "Anisotropy and size effects of surface roughness of rock joints," Chinese Journal of Rock Mechanics and Engineering, vol. 34, pp. 57-65, 2015.

[15] N. Fardin, O. Stephansson, and L. Jing, "The scale dependence of rock joint surface roughness," International Journal of Rock Mechanics and Mining Sciences, vol. 38, no. 5, pp. 659-669, 2001.

[16] R. Yong, J. Qin, M. Huang, S. Du, J. Liu, and G. Hu, "An innovative sampling method for determining the scale effect 
of rock joints," Rock Mechanics and Rock Engineering, pp. 1-12, 2018.

[17] F. Smarandache, Neutrosophy: Neutrosophic Probability, Set, and Logic, American Research Press, Rehoboth, Del, USA, 1998.

[18] F. Smarandache, Introduction to Neutrosophic Measure, Neutrosophic Integral, and Neutrosophic Probability, Sitech \& Education Publisher, 2013.

[19] F. Smarandache, Introduction to Neutrosophic Statistics, Sitech \& Education Publisher, 2014.

[20] J. Ye, "Bidirectional projection method for multiple attribute group decision making with neutrosophic numbers," Neural Computing and Applications, vol. 28, no. 5, pp. 1-9, 2015.

[21] J. Ye, "Multiple-attribute group decision-making method under a neutrosophic number environment," Journal of Intelligent Systems, vol. 25, no. 3, pp. 377-386, 2016.

[22] L. W. Kong, Y. F. Wu, and J. Ye, "Misfire fault diagnosis method of gasoline engines using the cosine similarity measure of neutrosophic numbers," Neutrosophic Sets and Systems, vol. 8, pp. 43-46, 2015.

[23] J. Ye, "Fault diagnoses of steam turbine using the exponential similarity measure of neutrosophic numbers," Journal of Intelligent \& Fuzzy Systems: Applications in Engineering and Technology, vol. 30, no. 4, pp. 1927-1934, 2016.

[24] F. Smarandache, Neutrosophic Precalculus and Neutrosophic Calculus, Europa-Nova, Brussels, 2015.

[25] J. Ye, R. Yong, Q.-F. Liang, M. Huang, and S.-G. Du, "Neutrosophic functions of the joint roughness coefficient (JRC) and the shear strength: a case study from the pyroclastic rock mass in Shaoxing City, China," Mathematical Problems in Engineering, vol. 2016, Article ID 4825709, 9 pages, 2016.

[26] R. Yong, J. Ye, B. Li, and S.-G. Du, "Determining the maximum sampling interval in rock joint roughness measurements using Fourier series," International Journal of Rock Mechanics and Mining Sciences, vol. 101, pp. 78-88, 2018.

[27] G. Zhang, M. Karakus, H. Tang, Y. Ge, and L. Zhang, "A new method estimating the 2D Joint Roughness Coefficient for discontinuity surfaces in rock masses," International Journal of Rock Mechanics and Mining Sciences, vol. 72, pp. 191-198, 2014.

[28] B. S. Tatone, Quantitative Characterization of Natural Rock Discontinuity Roughness In-Situ and in the Laboratory, Department of Civil Engineering, University of Toronto, 2009. 


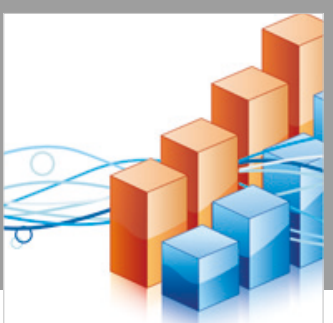

Advances in

Operations Research

\section{-n-m}
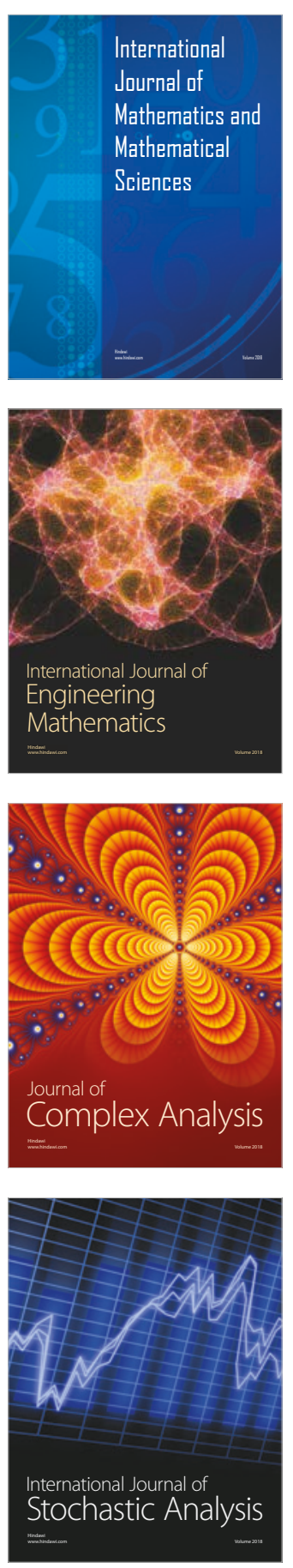
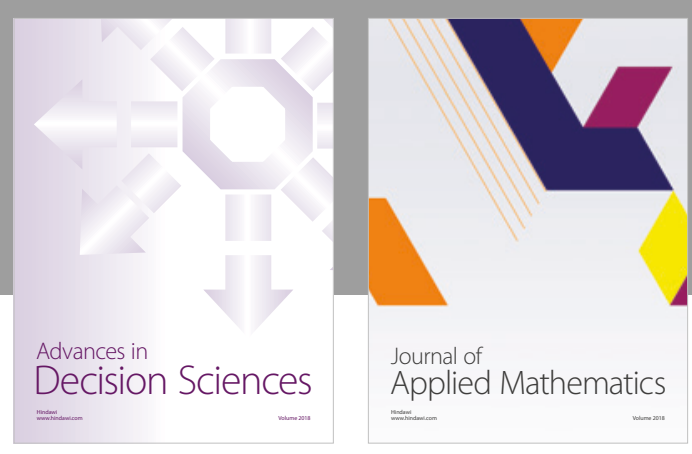

Journal of

Applied Mathematics
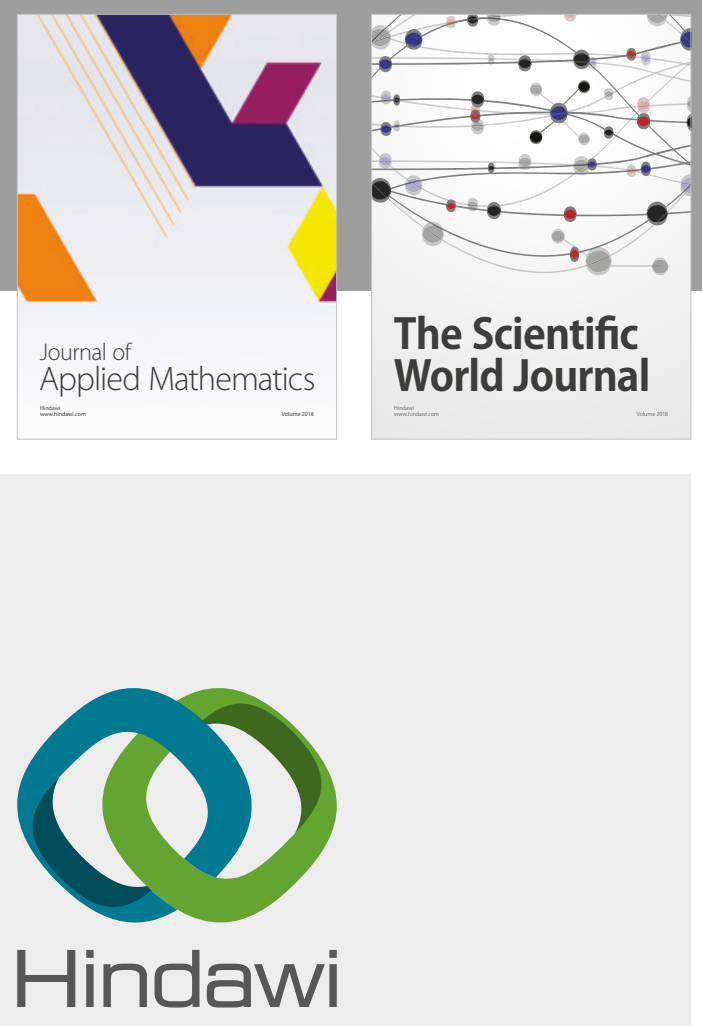

Submit your manuscripts at

www.hindawi.com

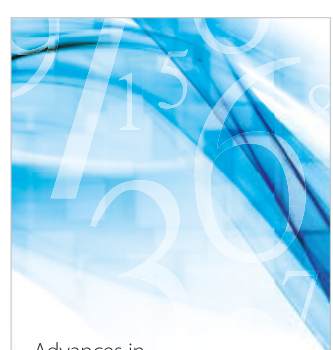

Advances in
Numerical Analysis
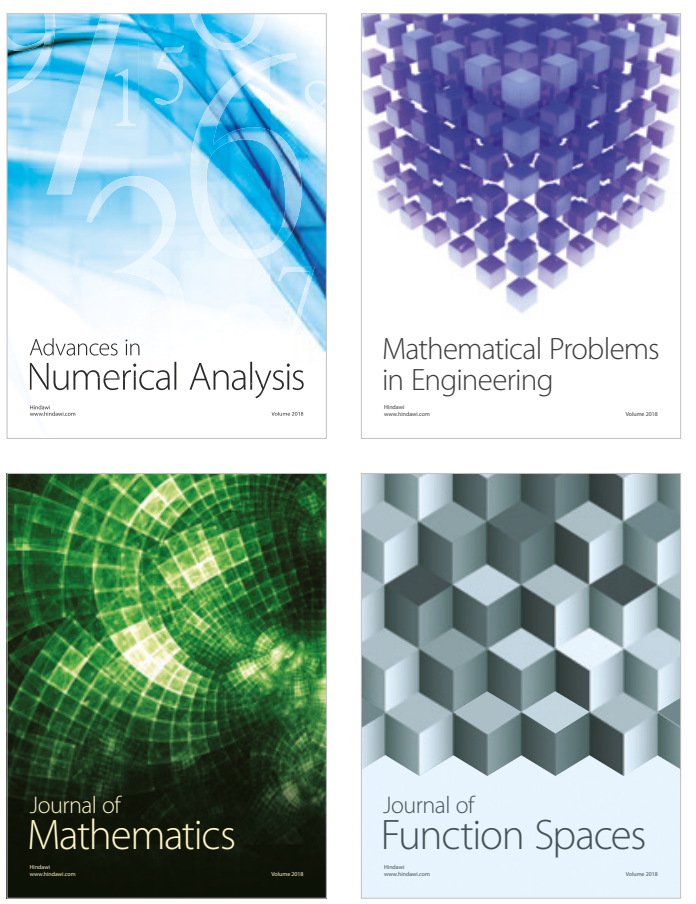

Mathematical Problems in Engineering

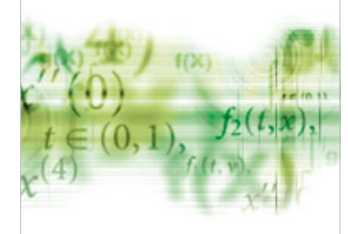

International Journal of

Differential Equations

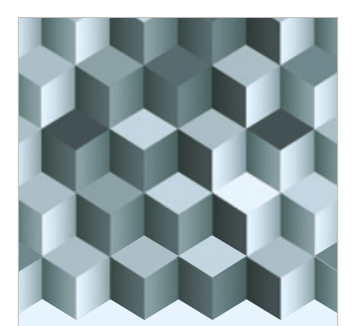

Journal of

Function Spaces

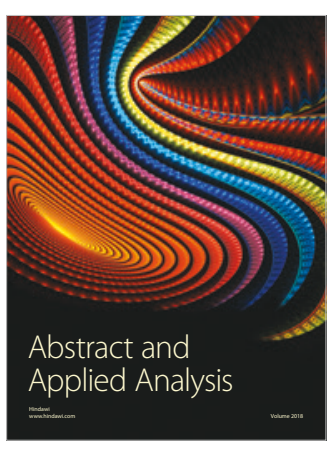

The Scientific

World Journal

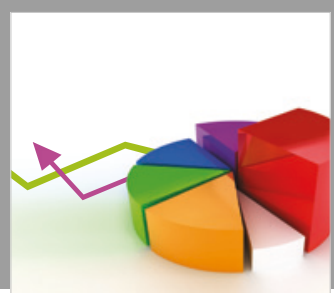

Journal of

Probability and Statistics
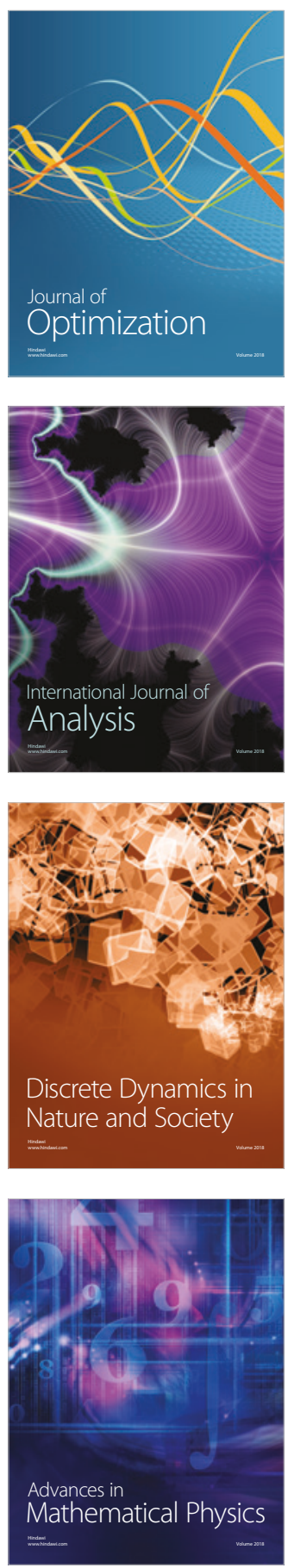